ЭКСПЕРИМЕНТАЛЬНЫЕ ИССЛЕДОВАНИЯ ДЕФОРМИРОВАНИЯ ЖЕЛЕЗОБЕТОННЫХ КОНСТРУКЦИЙ ПРИ КРУЧЕНИИ С ИЗГИБОМ

\author{
А.И. ДЕМЬЯНОВ*, к.т.н., дочент \\ Вл. И. КОЛЧУНОВ*, д.т.н., профессор \\ А.А. ПОКУСАЕВ $* *$, аспирант \\ *ФГБОУ ВО «Юго-Западный государственный университет», \\ 305040, Курск, ул.50 Лет Октября, 94; vlik52@таil.ru \\ **Московский государственный университет путей сообщения Императора \\ Николая II, Институт пути, строительства и сооружений, \\ 127055, Москва, Минаевский переулок,2,fvs_Aleksej456@yandex.ru
}

Приведены результаты экспериментальных исследований железобетонных конструкиий при сложном сопротивлении - кручении с изгибом, выполненные с целью выявления закономерностей и определения параметров деформирования и трещинообразования. Важнейшими из исследуемых параметров являлись: трещинообразующая нагрузка, разрушающая нагрузка, координаты образования пространственных трещин, ширина раскрытия этих трещин на уровне оси продольной и поперечной растянутой арматуры и вдоль всего профиля трещин; изменения расстояния между трещинами и длины трещин по мере увеличения нагрузки; деформации сжатого бетона и арматуры при сложном напряженно-деформированном состоянии. Результаты испытаний представлены в виде зависимостей прогибов конструкиий, деформаций сжатого бетона и рабочей арматуры, в том числе, определенных по показаниям электротензометрических розеток. Полученные зависимости позволяют оценивать достоверность разрабатываемых методов расчета железобетонных конструкиий при кручении с изгибом.

КЛЮЧЕВЫЕ СЛОВА: железобетон, кручение, изгиб, экспериментальные исследования, деформации, трещиностойкость, сложное сопротивление

Актуальность задачи. Процесс сложного сопротивления - кручения с изгибом в железобетоне представляет достаточно сложное явление, для моделирования которого требуется привлечение ряда исходных гипотез, подтвержденных экспериментом. Эта задача в теории железобетона до настоящего времени остается одной из наименее изученных в теоретическом плане и тем более в части экспериментальнойпроверки имеющихся [1-4] и создаваемых [5-9] расчетных моделей. В то же время, практическое использование железобетонных конструкций во все более сложных и ответственных зданиях и сооружениях сегодня вызывает необходимость развития теории и совершенствования методов расчета железобетонных конструкций при таких напряженных состояниях.

Уместно также заметить, что в любом конструктивном элементе, работающем на изгиб, может возникать кручение за счет случайного эксцентриситета, обусловленного внецентренным приложением вертикальной нагрузки, неоднородностью материалов неоднородностью материалов, асимметрией сечения.

На сегодняшний день, существует целый класс железобетонных конструкций (Г-образные рамы при действии ветровой нагрузки, опоры ЛЭП при одностороннем обрыве проводов, балки с боковыми консолями, наклонные арки и т.п.), в которых неучет кручения в расчетах может привести к обрушению конструкций, а в некоторых случаях обрушению и всего здания. Проведенные в зарубежных странах (США, Канаде и др.) ассоциацией портландцемента обследования зданий и сооружений выявили целый ряд случаев аварий, связанных с неверной оценкой сложного сопротивления железобетона. Неоспоримым явля- 
ется и тот факт, что сопротивление трещинообразованию пространственных трещин в железобетонных конструкциях само по себе явление достаточно сложное, а в условиях сложного кручения с изгибом - оно еще более усложняется.

Проведенные до настоящего времени лишь в единичных случаях и с ограниченным количеством изучаемых параметров экспериментальные исследования железобетонных конструкций при действии кручения с изгибом дают крайне ограниченную, а иногда и противоречивую информацию о их напряженно-деформированном состоянии, трещиностойкости, координатах пространственных трещин. Не изучены возникающие при этом эффекты, связанные с нарушением сплошности железобетона. Практически отсутствуют опытные данные о длине и приращениях трещин при увеличении ступени нагружения. В то же время, отмеченные другие параметры являются определяющими для анализа сопротивления областей, прилегающих к зонам пересечения трещинами рабочей арматуры, где, как показали последние исследования [4-6 и др.], возникает эффект нарушения сплошности бетона. Как показали опыты, влияние этого эффекта на равновесие усилий в поперечном сечении по результатам исследований проф. В.М. Бондаренко, Вл. И. Колчунова [4], может достигать 40\%.

Попытки разработки более совершенных расчетных моделей для решения задач образования различных типов пространственных трещин на нижней и боковой грани железобетонных конструкций при кручении с изгибом были сделаны в работах [6-10]. В развитие этих исследований, в настоящей работе приведены методика и результаты экспериментальных исследований для проверки предложенных расчетных моделей железобетона.

Методика исследований. Проведенные экспериментальные исследования были направлены на определение основных параметров железобетонных конструкций при изгибе с кручением: нагрузки образования пространственных трещин $R_{\text {sup,crc }}$, разрушающей нагрузки $R_{\text {sup }, u}$, координат образования пространственных трещин, фактической высоты сжатой зоны бетона $x_{\text {fact }}$ в поперечном сечении, проходящим через конец пространственной трещины; прогибы конструкций; ширина раскрытия трещин на уровне оси продольной и поперечной растянутой арматуры вдоль всего профиля трещин; изменения расстояния между трещинами $l_{c r c}$ и длины трещин $h_{c r c}$ по мере увеличения нагрузки и др.

Измерение названных опытных характеристик проводилось механическими, оптическими и тензометрическими приборами, устанавливаемыми на опытных конструкциях. В частности, для измерения перемещений опытных конструкций использовались прогибомеры с ценой деления 0,01 мм (рис. 1). Деформации арматуры измерялись специальными устройствами, включавшими гайки, приваренные к рабочей арматуре, на которые перед бетонированием балок, одевались специальные резиновые трубки, а торцы гаек закрывались пластилином с целью исключения влияния окружающего бетона на положение «гаек» при деформациях. При измерении деформаций арматуры в эти гайки вкручивались штанги, на которые крепились индикаторы с ценой деления 0,001 мм на базе 250 мм.

Деформации бетона измерялись отдельными тензорезисторами и розетками тензорезисторов. В процессе кратковременного нагружения отсчеты по тензорезисторам и по механическим приборам снимались дважды: сразу после приложения нагрузки и после временной выдержки.

С помощью микроскопа МПБ-2 с 24-х кратным увеличением и ценой деления 0,05 мм изучалась растянутая зона каждой конструкции. Появление и дальнейшее распространение трещин тщательно фиксировалось на специальных 
планшетах. По мере дальнейшего нагружения проводилось наблюдение за появлением новых трещин и развитием уже имеющихся. Замерялась ширина раскрытия трещин на двух боковых гранях в уровнях, расположенных выше оси арматуры на 20 и 30 мм.

Методика экспериментальных исследований предусматривала установкучетырех групп электротензорезисторов на опытные конструкции. Электротензо-
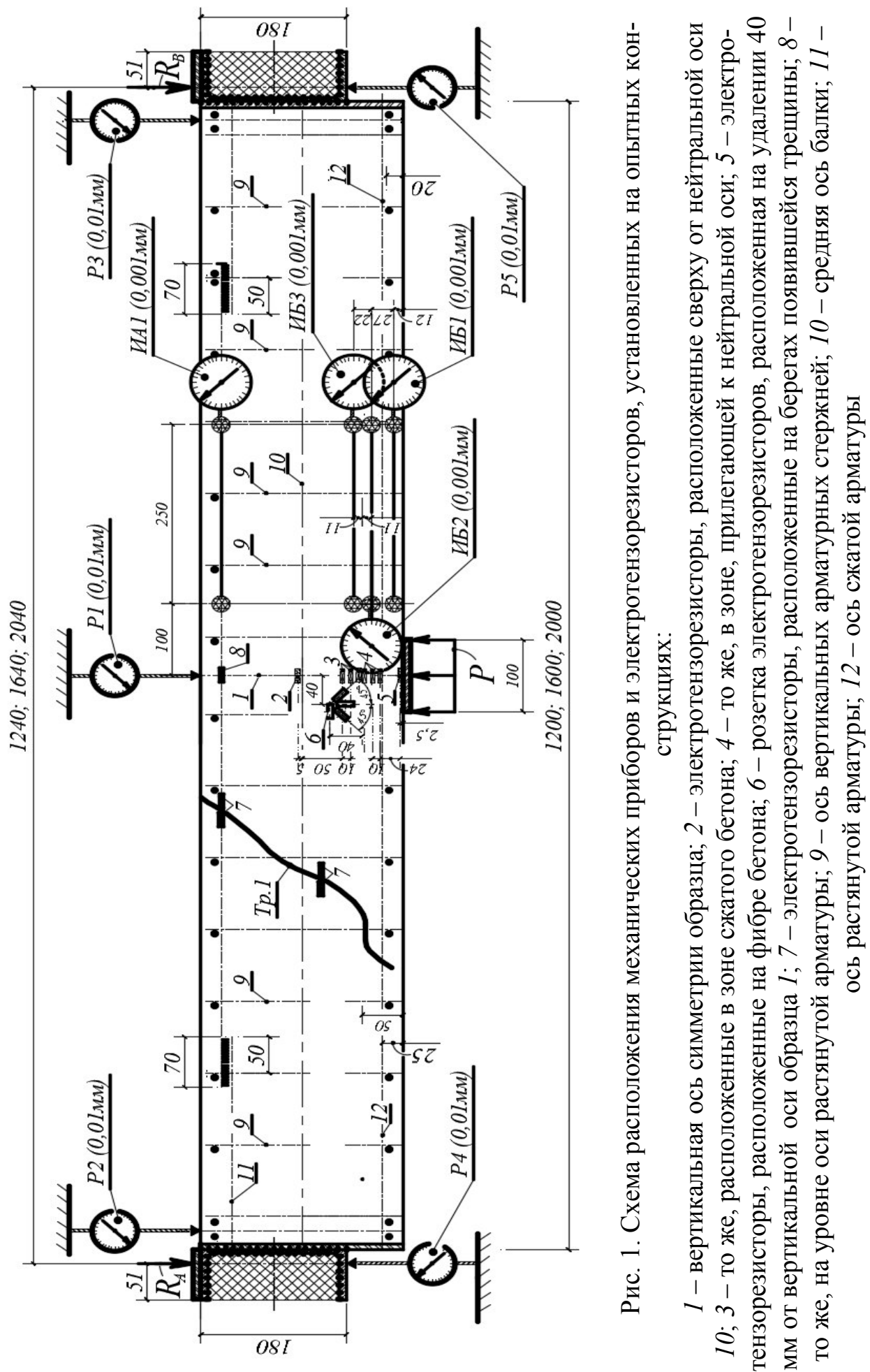
резисторы первой группы (см. рис. 1) устанавливались по высоте поперечного сечения экспериментальных конструкций основных серий в фибровых волокнах, в местах расположения нулевой точки, т. е. перехода от удлинения к укорочению.

Изучение распределения деформаций по всей высоте сжатой зоны, определение размеров сжатой зоны и фактической высоты развития трещин стало возможным благодаря установке электротензорезисторов, цепочкой по высоте образцов. Обеспечение надежности и дополнение опытной информации осуществлялось установкой на опытных конструкциях групп розеток, располагаемых в двух одинаково напряженных симметричных относительно вертикальной оси балки сечениях. Электротензорезисторы второй группы устанавливались на берегах, образующихся при нагружении трещин на уровне оси растянутой арматуры.

Для повышения надежности экспериментальных данных предусматривалось дублирование работы электротензорезисторов (там, где это было возможно) механическими приборами - индикаторами с ценой деления 0,001мм, устанавливаемыми на базе 250 мм (см. рис. 1).

Конструкции опытных образцов, их маркировка, схемы армирования и схемы нагружения были подробно описаны в работе [11].

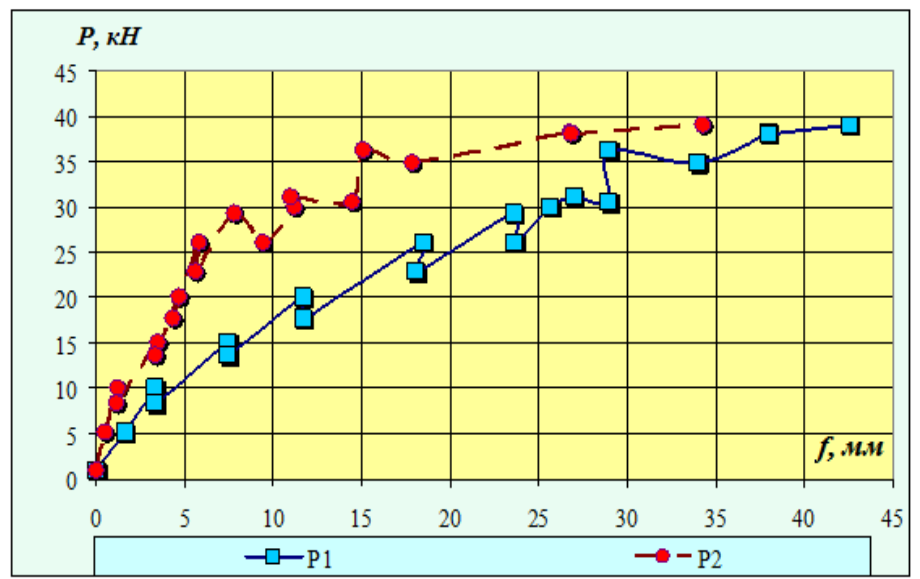

Рис. 2. Зависимости «нагрузка- прогиб» в опытной конструкции балки КИП-II-1,6

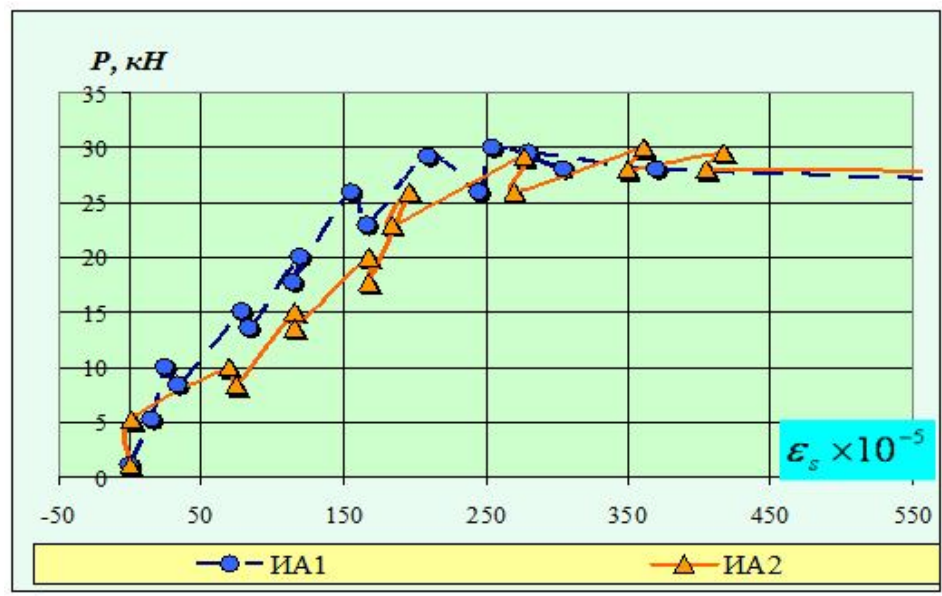

Рис. 3. Зависимости «нагрузка-деформация» рабочей арматуры опытной конструкции КИП-II-1,6 
Результаты исследований. Полученные опытные данные о деформировании железобетонных балок при кручении с изгибом позволяют отметить следующее.

Прогибы опытных балок шифра КИП-ІІ-1,6 (К-кручение, И-изгиб, П - прямоугольное сечение, 1,6 - длина конструкции в метрах) с относительно невысоким уровнем нагружения имели ярко выраженный нелинейный характер (рис. 2). При этом по графикам деформаций рабочей арматуры (рис. 3) не сложно проследить начало и развитие трещинообразования в конструкциях балок.

Были получены также опытные значения деформаций бетона по тензорезисторам и розеткам тензорезисторов установленным по высоте среднего поперечного сечения опытных конструкций. Графики деформаций полученных по конкретным тензорезисторам (R5-R8) на различных этапах нагружения для одной из опытных конструкции (КИП-ІІ-1,6) приведены на рис. 4.

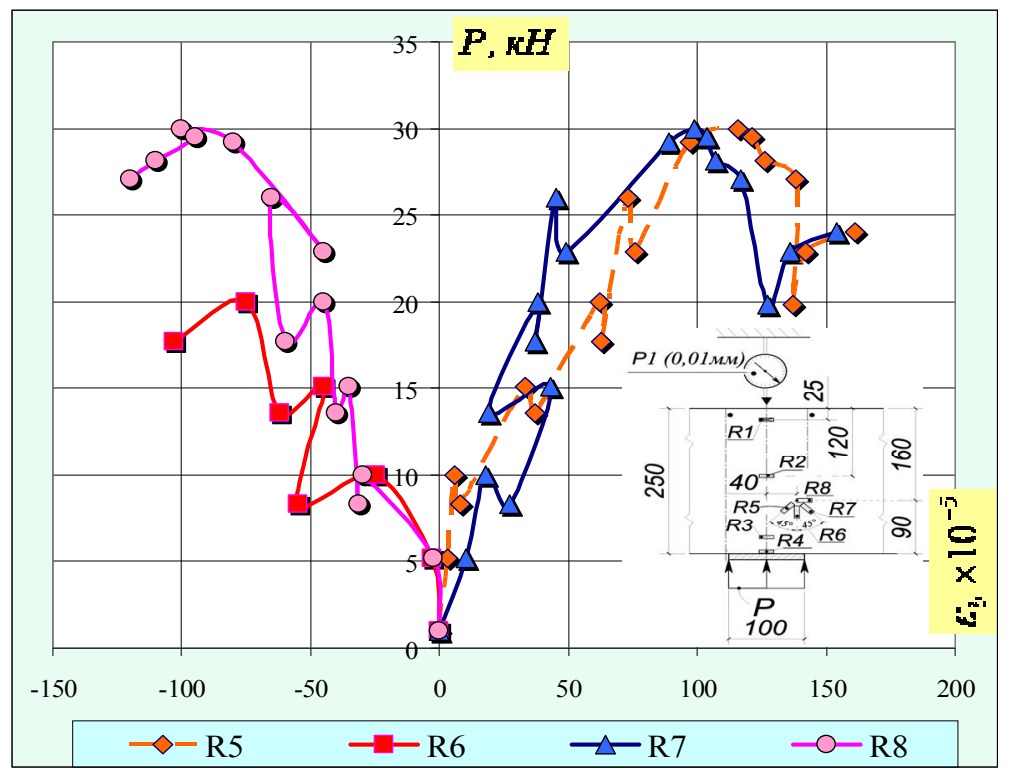

Рис. 4. Графики зависимости деформаций бетона по показаниям розетки тензорезисторов в опытной конструкции КИП-II-1,6

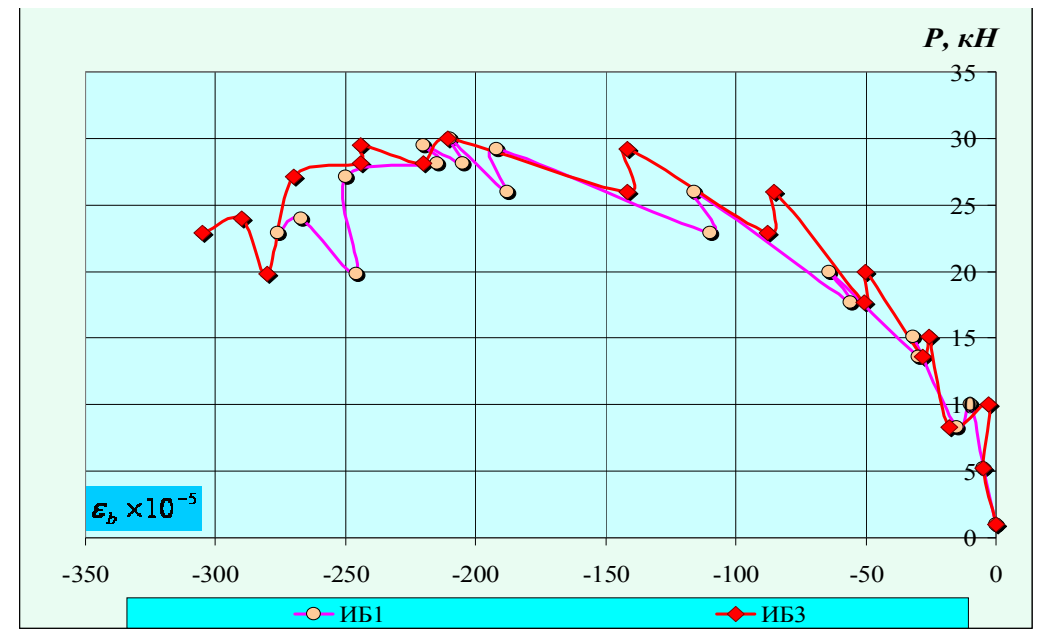

Рис. 5. Графики зависимости опытных деформаций бетона в опытной конструкции КИП-II-1,6 
Помимо тензометрии, достоверность этих деформаций контролировались механическими приборами (индикаторами, ИБ, см. рис. 1), результаты измерений, которые приведены на рис.5. Не сложно видеть, что в диапазоне деформаций $\varepsilon=0-100 \cdot 10^{-5}$ имеется удовлетворительное количественное согласование измеренных значений деформаций по показаниям тензорезистров и индикаторов.

Экспериментальными исследованиями были определены и ряд других параметров характеризующих особенности деформирования и трещинообразования железобетонных конструкций при кручении с изгибом, в их числе координаты образования пространственных трещин и ширина их раскрытия, изменения расстояния между трещинами и длины трещин в процессе нагружения, и ряд других. Эти данные планируется представить в последующих публикациях авторов.

Полученные экспериментальные зависимости дают достаточно полное представление о закономерностях деформирования и предельных значениях нагрузки для сложно напряженных железобетонных конструкций при кручении с изгибом.

Вывод. Предложенная методика экспериментальных исследований и полученные результаты испытаний опытных конструкций железобетонных балок при сложном сопротивлении дают возможность проверки достоверности рабочих гипотез и разрабатываемых расчетных моделей деформирования железобетонных конструкций при кручении с изгибом.

(C) Демьянов А.И., Колчунов Вл.И., Покусаев А.А. 2017

Список литературы

1. Верюжский Ю.В., Гольшев А.Б., Колчунов Вл. И., Клюева Н.В., Лисицин Б.М., Машков И.Л., Яковенко И.А. Справочное пособие по строительной механике. В двух томах. Том I: Учебное пособие. - М.: Издательство АСВ, 2014. - 640 с.

2. Голышев А.Б. Сопротивление железобетона [Текст]: монография / А.Б. Голышев, Вл. И. Колчунов. - К.: Основа, 2009. - 432 с.

3. Карпенко Н.Н., Елагин Э.Г. Деформации железобетонных трубчатых элементов при изгибе с кручением// Прочность и жесткость железобетонных конструкций. - М.: НИИЖБ Госстроя СССР, 1971. - С. 29-48.

4. Бондаренко В.М., Колчунов В.И. Расчетные модели силового сопротивления железобетона: монография. М.: АСВ, 2004. - 472c.

5. Клюева Н.В., Яковенко И.А., Усенко Н.В. К расчету ширины раскрытия наклонных трещин третьего типа в составных железобетонных конструкциях // Промышленное и гражданское строительство. 2014. № 2. - С. 8-11.

6. Salnikov A., Kolchunov Vl., Yakovenko I. The computational model of spatial formation of cracks in reinforced concrete constructions in torsion with bending (2015), Applied Mechanics and Materials. Vols. 725-726 (2015), pp. 784-789.

7. Сальников А.С., Колчунов Вл.И., Яковенко И.А. Расчетная модель образования пространственных трещин первого вида при кручении с изгибом // Промышленное и гражданское строительство. 2015. №3. - С. 35-40.

8. Сальников А.С., Колчунов Вл. И., Колчунов В. И. Методика расчета предельной нагрузки и координат образования пространственной трещины первого вида в железобетонных конструкциях при кручении с изгибом // Строительство и реконструкция. 2015. №6(62). - C. 49- 56.

9. Колчунов Вл.И., Рыпаков Д.А. Моделирование пространственной трещины в железобетонных конструкциях при кручении с изгибом // Строительная механика инженерных конструкций и сооружений. 2016. № 5. - С. 11-16.

10. Сальников А.С., Клюева Н.В., Колчунов Вл.И. Метод определения минимальной нагрузки и координат образования пространственной трещины в железобетонных кон- 
струкциях при кручении с изгибом // Промышленное и гражданское строительство. 2016. № 1. - С. 52-57.

11. Колчунов Вл.И., Сальников А.С. Экспериментальные исследования трещинообразования в железобетонных конструкциях при кручении с изгибом// Строительство и реконструкция. 2016. №3(65). - С. 24-32.

Поступила в редакцию 2 июня 2017 г. Прошла рецензирование 12 октября 2017 г. Принята к публикаичи 18 октября 2017 г.

\section{Об авторах:}

ДЕМЬЯНОВ АЛЕКСЕЙ ИВАНОВИЧ, к.т.н., дочент кафедры "Промыиленное и гражданское строительство, ФГБОУ ВО «Юго-Западный государственный университет». Научные интересы: исследования железобетонных конструкиий при сложном сопротивлении, конструктивная безопасность строительных систем из железобетона и других нелинейно деформированных материалов, экспериментальные исследования железобетонных конструкиий, компьютерные технологии расчетного анализа зданий и сооружений, 305040, Курск, ул. 50 Лет Октября, 94; speccompany@gmail.com

КОЛЧУНОВ Владимир Иванович, д.т.н., профессор кафедры «Промышленное и гражданское строительство», ФГБОУ ВО «Юго-Западный государственный университет». Научные интересы: строительная механика, строительные конструкции, теория железобетона, экспериментальные исследования строительных конструкиий, физические и математические модели механики сооружений,305040, Курск, ул. 50 Лет Октября, 94; vlik52@mail.ru

ПОКУСАЕВ Алексей Александрович, аспирант, Московский государственный университет путей сообщения Императора Николая II, Институт пути, строительства и сооружений. Научные интересы: теоретические и экспериментальные исследования конструкций зданий и сооружений, моделирование железобетонных конструкиий, испытания железобетонных конструкиий с элементами сплошного и составного сечения, 127055, Москва, Минаевский переулок, 2,fvs_Aleksej456@yandex.ru

\section{Для цитирования:}

Демьянов А.И., Колчунов Вл.И., Покусаев А.А. Экспериментальные исследования деформирования железобетонных конструкций при кручении с изгибом // Строительная механика инженерных конструкций и сооружений. - 2017. - № 6. - C. 37-44. Doi: 10.22363/1815-5235-2017-6-37-44

\section{References}

1. Veryuzhskiy,Yu.V., Golyshev, A.B., Kolchunov, Vl.I., Klyueva, N.V., Lisitsin, B.M., Mashkov, I.L., Yakovenko, I.A. (2014). Spravochnoe posobie postroitel'noy mekhanike [Reference Manualon Building Mechanics], Tom I: Uchebnoe posobie, ASV Publ., Moscow. 640 p. (In Russ.)

2. Golyshev, A.B. (2009). Soprotivlenie Zhelezobetona [Resistance of Reinforced Concrete]: monography, Osnova Publ., Kiev. 432 p. (In Russ.)

3. Karpenko, N.N., Elagin, E.G. (1971). Deformatsii Zhelezobetonnykh Trubchatykh Elementov pri Izgibe s Krucheniem. Prochnost' $i$ Zhestkost' Zhelezobetonnykh Konstruktsiy [Durability and rigidity of reinforced concrete structures]. NIIZhB Gosstroya SSSR, Moscow. P. 29-48. (In Russ.)

4. Bondarenko, V.M., Kolchunov, V.I. (2004), Raschetnye Modeli Silovogo Soprotivleniya Zhelezobetona [The computational model of power resistance of reinforced concrete], ASV Publ. Moscow. 472 p. (In Russ.)

5. Klueva, N.V., Yakovenko, I.A., Usenko, N.V. (2014). K raschetu Shiriny Raskrytiya Naklonnykh Treshchin Tret'ego Tipa v Sostavnykh Zhelezobetonnykh konstruktsiyakh, Promyshlennoe $i$ Grazhdanskoe Stroitel'stvo [Industrial and Civil Engineering]. No. 2. P. 8-11. (In Russ.)

6. Salnikov, A., Kolchunov, Vl., Yakovenko, I. (2015). The computational model of spatial formation of cracks in reinforced concrete constructions in torsion with bending. Applied Mechanics and Materials. Vols. 725-726. P. $784-789$.

7. Salnikov, A.S., Kolchunov, Vl.I., Yakovenko, I.A. (2015). Raschetnaya model' obrazovaniya prostranstvennykh treshchin pervogo vida pri kruchenii s izgibom. Promyshlennoe $i$ Grazhdanskoe stroitel'stvo [Industrial and Civil Engineering]. No 3. P. 35-40. (In Russ.)

8. Salnikov, A.S., Kolchunov, Vl.I., Kolchunov, V.I. (2015). Metodika rascheta predel'noy nagruzki i koordinat obrazovaniya prostranstvennoy treshchiny pervogo vida $\mathrm{v}$ zhelezobetonnykh konstruktsiyakh pri kruchenii s izgibom. Building \& Reconstruction. No 6(62). P. 49-56. (In Russ.)

9. Kolchunov, Vl.I., Rypakov, D.A. (2016). Modeling of cracks in reinforced concrete constructions in torsion with bending. Structural Mechanics of Engineering Constructions and Buildings. No 5. P.1116. (In Russ.) 
10. Salnikov, A.S., Klueva, N.V., Kolchunov, Vl.I. (2016). The method for determining minimum load and the coordinates of the spatial crack formation in reinforced concrete structures under torsion with bending. Promyshlennoe i Grazhdanskoe Stroitel'stvo [Industrial and Civil Engineering]. Vol. 1. P. 52-57. (In Russ.)

11. Kolchunov, Vl.I., Sal'nikov, A.S. (2016). Experimental research of crack appearance in reinforced concrete structures under torsion with bending. Building \& Reconstruction. No 3(65). P. $24-32$.

\title{
EXPERIMENTAL STUDIES OF DEFORMATION OF REINFORCED CONCRETE STRUCTURES SUBJECTED TORSION AND BENDING
}

\author{
A.I. DEMYANOV*, Vl.I. KOLCHUNOV*, A.A. POKUSAEV** \\ "Southwest state university, Kursk, Russia \\ *** Moscow State University of Railway Engineering (MIIT), \\ **Institute of Railways, Construction and Structures, Russia
}

The results of experimental studies of reinforced concrete constructions at complex resistance to torsion with bending are shown in the article. The main purposes of the researches are detection of regularities and determination of deformation and cracking parameters. The most important of the studding parameters are cracking load, destructive load, the coordinates of spatial cracks formation, the width of crack's opening at the axis of the longitudinal and transverse tensile reinforcement and along the entire profile of cracks; changing the distance between the cracks and crack length according to increasing the deformation load; deformation of compressed concrete and reinforcement in a complex stress-strain state. The test results are presented in the form of relationships of structure's deflections, deformations of compressed concrete and reinforcement, including results which were determined by the indications of electrotensometric sockets. The obtained relationships allow us to assess the reliability of the calculating methods reinforced concrete constructions subjected the action torsion and bending.

Keywor d s : reinforced concrete, torsion, bending, experimental studies, deformations, crack resistance, complex stress state.

Article history: Received: June 2, 2017. Revised: October 12, $2017 . \quad$ Accepted: October 18, 2017.

\section{About the authors:}

DEMYANOV ALEKSEJ IVANOVICH, candidate of technical sciences, associate professor of the department of industrial and civil engineering. Scientific interests: the study of composite structures in complex resistance, the study of the survivability of structural systems and reinforced concrete and other non-linearly deformed materials, experimental studies in reinforced concrete structures, computer technologies for computational analysis of buildings and structures, South-West State University, 305040, Kursk, St.50 Years of October, speccompany@gmail.com

KOLCHUNOV VLADIMIR IVANOVICH, doctor of technical sciences, Professor of the department of industrial and civil engineering. Scientific interests: building mechanics, building structures, reinforced concrete theory, experimental studies of building structures, physical and mathematical models of structures, 305040, Kursk, St.50 Years of October, vlik52@mail.ru

POKUSAEV ALEXEI ALEXANDROVICH, graduate student, Moscow State University of Communications, Emperor Nicholas II, Institute of Ways, Construction and Structures. Scientific interests: theoretical and experimental studies of the survivability of buildings and structures, modeling of reinforced concrete structures, testing of reinforced concrete structures with solid and composite element, 127055, Moscow, Minaevsky pereulok, 2, fvs_Aleksej456@yandex.ru

\section{For citation:}

Demyanov A.I., Kolchunov Vl.I., Pokusaev A.A. (2017) Experimental studies of deformation of reinforced concrete structures subjected torsion and bending. Structural Mechanics of Engineering Constructions and Buildings. No 6. 37-44. Doi: 10.22363/1815-5235-2017-6-37-44. (In Russ.) 